\title{
MOLECULAR GAS IN HIGH REDSHIFT QSOS
}

\author{
C. L. CARILLI ${ }^{1}$, F. BERTOLDI ${ }^{2}$, F. WALTER ${ }^{1}$, K.M. MENTEN ${ }^{2}$, A. \\ BEELEN $^{3}$, P. COX ${ }^{3}$, A. OMONT ${ }^{4}$ \\ ${ }^{1}$ National Radio Astronomy Observatory, ${ }^{2}$ Max-Planck Inst. for Radio \\ Astronomy, ${ }^{3}$ Univ. Paris-Sud, ${ }^{4}$ Inst. d'Astrophysique de Paris \\ E-mail: ccarilli@nrao.edu
}

\begin{abstract}
We review $\mathrm{cm}$ and $\mathrm{mm}$ observations of thermal molecular line emission from high redshift QSOs. These observations reveal the massive gas reservoirs $\left(10^{10}\right.$ to $10^{11}$ $\mathrm{M}_{\odot}$ ) required to fuel star formation at high rates. We discuss evidence for active star formation in QSO host galaxies, and we show that these high redshift, FIR-luminous QSOs follow the non-linear trend of increasing $\mathrm{L}_{F I R} / \mathrm{L}^{\prime}(\mathrm{CO})$ with increasing $\mathrm{L}_{F I R}$. We conclude with a brief discussion of the recent $\mathrm{CO}$ detection of the most distant QSO at $z=6.42$, and its implications for cosmic reionization.
\end{abstract}

\section{Introduction}

Over the last few years, the study of high redshift QSOs has been revolutionized in three ways. First, wide field surveys have revealed 100's of high z QSOs, right back to the epoch of cosmic reionization $(z>6$; e.g., Fan et al. 2003). Second, it has been shown that most (all?) low redshift spheroidal galaxies have central super-massive black holes (SMBH), and that the black hole mass correlates with bulge velocity dispersion. This $\mathrm{M}_{B H^{-}} \sigma_{\mathrm{v}}$ correlation suggests coeval formation of galaxies and $\mathrm{SMBH}$, thereby making SMBHs a fundamental aspect of the galaxy formation process (Gebhardt et al. 2000). And third, mm surveys of high redshift QSOs find that 30\% of the sources are 'hyper-luminous infrared galaxies' $\left(L_{F I R}=10^{13} \mathrm{~L}_{\odot}\right)$, corresponding to thermal emission from warm dust, and that this fraction is independent of redshift out to $z=6.4$ (Omont et al. this vol.). If the dust is heated by star formation, the implied star formation rates are extreme $\left(>10^{3} \mathrm{M}_{\odot}\right.$ year $\left.^{-1}\right)$, consistent with the formation of a large elliptical galaxy on a dynamical timescale of $10^{8}$ years. On the other hand, the FIR

*the national radio astronomy observatory is a facility of the national science foundation operated under cooperative agreement by associated universities, inc. 
luminosity constitutes typically only $10 \%$ of the bolometric luminosity of the sources, such that dust heating by the AGN remains an alternative. Demographic studies show that SMBHs acquire most of their mass during major accretion events marked by the QSO phenomenon (Yu \& Tremain 2002).

Molecular line observations (typically CO) of FIR-luminous high $z$ QSOs have revealed large gas masses in most cases observed to date (see Table 1). Such gas reservoirs are a prerequisite for star formation models for dust heating in FIR-luminous high $z$ QSOs. The typical gas depletion timescales are of order $10^{7}$ to $10^{8}$ years, if the dust is heated by star formation. In this review we consider this question in more detail. We restrict ourselves to $z>2$ QSOs (see also Barvainis 1999; and see Scoville et al. 2004 and Sanders \& Mirabel 1996 for observations of lower redshift QSOs). We assume a standard concordance cosmology.

\section{Statistics}

Table 1 shows all the published detections of $\mathrm{CO}$ emission from sources at $z>2$ (only the most recent reference is given). Most of the sources are AGN (QSOs, radio galaxies $=\mathrm{RG}$ ), since in these cases optical spectroscopic redshifts are available, although a number of optically brighter 'submm galaxies' (SMM) have now been detected in CO emission (Neri et al. 2003). About half the sources are strongly lensed (L). Most sources have been studied in the higher order transitions ( $\geq \mathrm{CO} 3-2$ ), although at $z \geq 4$ the lower order transitions become accessible to $\mathrm{cm}$ telescopes such as the VLA.

Figure 1 shows the correlation between FIR luminosity and velocity integrated CO 1-0 luminosity $\left(L_{C O(1-0)}^{\prime} \mathrm{K} \mathrm{km} \mathrm{s}^{-1} \mathrm{pc}^{2}\right)$ for sources at low and high redshift (Beelen et al. 2004). The sources in Table 1 consitute most of the sources with $L_{F I R}>10^{13} \mathrm{~L}_{\odot}$ in Figure 1. For sources without CO 1-0 measurements, the 1-0 luminosity was calculated assuming constant brightness temperature. For the high redshift sources the FIR luminosity is given approximately by: $L_{F I R}=4 \times 10^{12}\left(S_{250} / \mathrm{mJy}\right) \mathrm{L}_{\odot}$, appropriate for a typical ULIRG SED (Omont et al. al. 2003; note that in Fig 1 the values were calculated using the measured multifrequency SEDs where available). For dust heating by star formation, the total star formation rate (SFR; from 0.1 to $100 \mathrm{M}_{\odot}$ ) is given by: $S F R=4 \times 10^{-10} L_{F I R} \mathrm{M}_{\odot}$ year $^{-1}$. Gas masses can be derived from: $\mathrm{M}\left(\mathrm{H}_{2}\right)=X \times L_{C O(1-0)}^{\prime}$, where $X=4$ for typical spiral galaxies, and $X=0.8$ for ULIRGs (Downes \& Solomon 1998). Note that $X=0.2$ is the minimum (ie. optically thin) value, assuming solar $\mathrm{C}$ and $\mathrm{O}$ 
Table 1. CO sources at $z>2$ as of February 2004

\begin{tabular}{lcccccl}
\hline name & type & $z$ & Trans. & $\begin{array}{c}\text { L' } \\
\text { Jy km/s }\end{array}$ & $\begin{array}{c}\text { S250 } \\
\text { mJy }\end{array}$ & ref \\
\hline B1021+4724 & QSOL & 2.286 & $3-2$ & 4.2 & 9.6 & Solomon 1992 \\
J1636+4057 & SMM & 2.385 & $3-2$ & 2.3 & 2.5 & Neri 2003 \\
53 W02 & QSO & 2.394 & $3-2$ & 1.2 & 1.7 & Alloin 2000 \\
J0443+0210 & SMM & 2.509 & $3-2$ & 1.4 & 1.1 & Neri 2003 \\
Cloverleaf & QSOL & 2.558 & $3-2$ & 9.9 & 18 & Weiss 2004 \\
J1401+0252 & SMML & 2.565 & $3-2$ & 2.4 & & Frayer 1999 \\
J1409+5628 & QSO & 2.583 & $3-2$ & 4.0 & 10.7 & Beelen 2004 \\
B0414-0534 & QSOL & 2.639 & $3-2$ & 2.6 & 40 & Barvainis 1998 \\
cB58 & SMML & 2.727 & $3-2$ & 0.3 & 1 & Baker 2003 \\
J1230+1627 & QSOL & 2.735 & $3-2$ & 0.80 & 2.7 & Guilloteau 1999 \\
J0239-0136 & SMML & 2.808 & $3-2$ & 3.1 & 4.0 & Genzel 2003 \\
J2330+3927 & RG & 3.094 & $4-3$ & 1.3 & & de Breuck 2003 \\
B0751+2716 & QSOL & 3.200 & $4-3$ & 6.0 & 6.7 & Barvainis 2002 \\
J0943+4700 & SMM & 3.346 & $4-3$ & 1.1 & 2.3 & Neri 2003 \\
J0121+1320 & RG & 3.520 & $4-3$ & 1.2 & & de Breuck 2003 \\
J1909+722 & RG & 3.534 & $4-3$ & 1.6 & 4 & Papadopoulos 2000 \\
4 C60.07 & RG & 3.788 & $1-0$ & 0.24 & 4.5 & Greve 2004 \\
B0827+5255 & QSOL & 3.911 & $1-0$ & 0.15 & 17.0 & Papadopoulos 2001 \\
J2322+1944 & QSOL & 4.119 & $2-1$ & 0.92 & 9.6 & Carilli 2003 Cox 2002 \\
B0952-0115 & QSOL & 4.434 & $5-4$ & 0.91 & 2.8 & Guilloteau 1999 \\
B1335-0417 & QSO & 4.407 & $2-1$ & 0.44 & 5.6 & Carilli 2002 \\
B1202-0725 & QSO & 4.693 & $2-1$ & 0.49 & 12.6 & Carilli 2002 \\
J1148+5251 & QSO & 6.419 & $3-2$ & 0.20 & 5.0 & Walter 2003 Bertoldi 2004 \\
\hline
\end{tabular}

abundances, and warm gas $(70 \mathrm{~K})$.

The straight lines correspond to linear relations, with a ratio of $L_{F I R} / L_{C O}^{\prime}=40$ (dash) or 300 (solid). The correlation is clearly nonlinear, and is better described by a power-law of the form: $L_{F I R} \propto L_{C O}^{\prime}{ }^{1.7}$. This non-linearity has been interpreted as an increasing 'star formation efficiency' (= SFR/gas mass) with increasing SFR (Gao \& Solomon 2003). An alternate hypothesis is that the AGN dominates dust heating at the highest luminosities. But this latter hypothesis begs the question: why would there be any correlation at all? Meaning that if two very different physical mechanisms are involved, one might expect a discontinuity in this relationship going from starbursts to AGN.

\section{Examples}

Figure 2 shows examples of the CO excitation for three $z>4$ QSOs. The line strengths are consistent with constant brightness temperature $\left(\mathrm{T}_{B}\right)$ up to CO 5-4. This is similar to what is seen for nuclear starburst regions, such as in M82, and implies dense $\left(>10^{4} \mathrm{~cm}^{-3}\right)$, warm $(>50 \mathrm{~K})$ gas. The recent detection of $\mathrm{HCN}$ emission from the Cloverleaf quasar at $z=2.56$ 


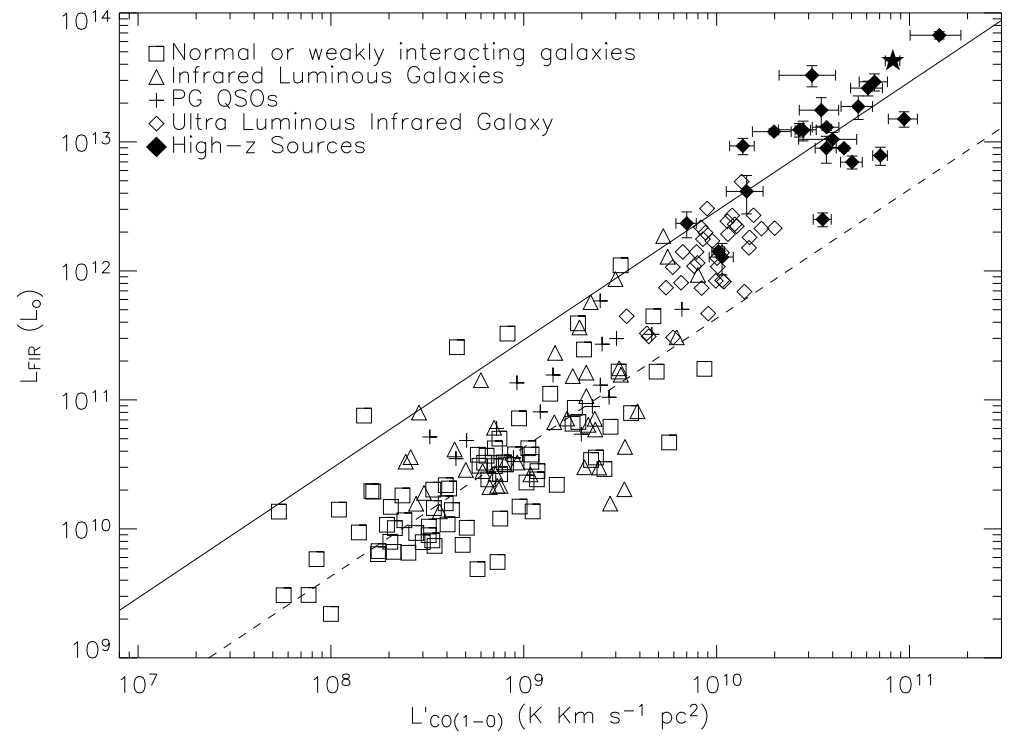

Figure 1. The relationship between $L_{F I R}$ and $L_{C O(1-0)}^{\prime}$ (Beelen et al. 2004).

supports the idea of dense, warm (star forming?) molecular gas in these systems (Solomon et al. 2003).
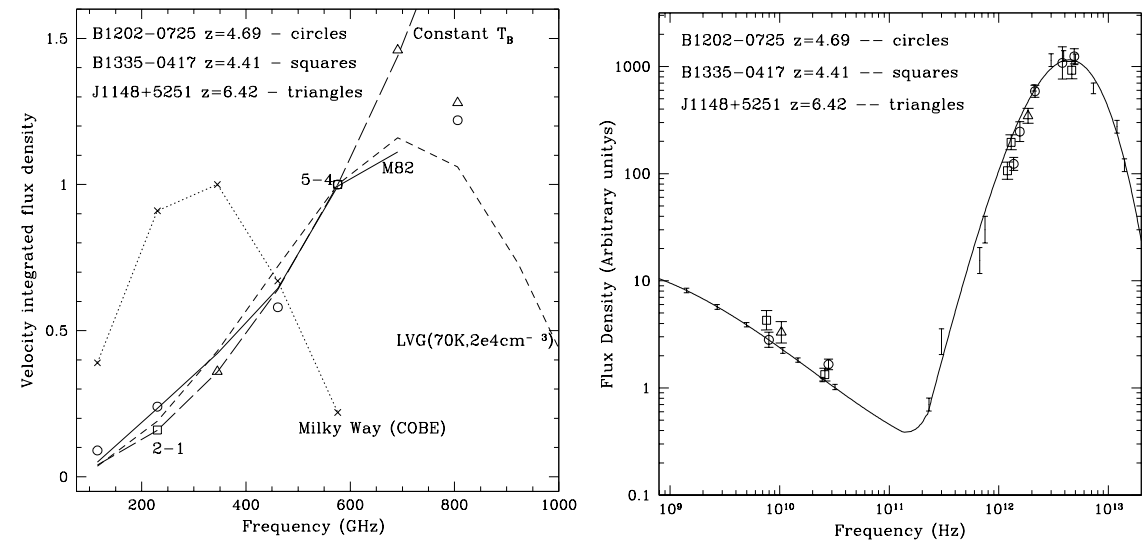

Figure 2. Left: The CO ladder for three high $z$ QSOs. Right: The radio to IR SEDs. The solid line is the fit for M82. 
Also shown in Figure 2 are the radio to IR SEDs of these sources. Again, the SEDs are similar to those seen in typical star forming galaxies. VLBA imaging of the $1.4 \mathrm{GHz}$ continuum emission from $\mathrm{J} 1409+5628$ at $z=2.58$ implies intrinsic brightness temperatures $<10^{5} \mathrm{~K}$, consistent with the nonthermal emission expected from a star forming galaxy, but inconsistent with that expected from an AGN (Beelen et al. 2004).
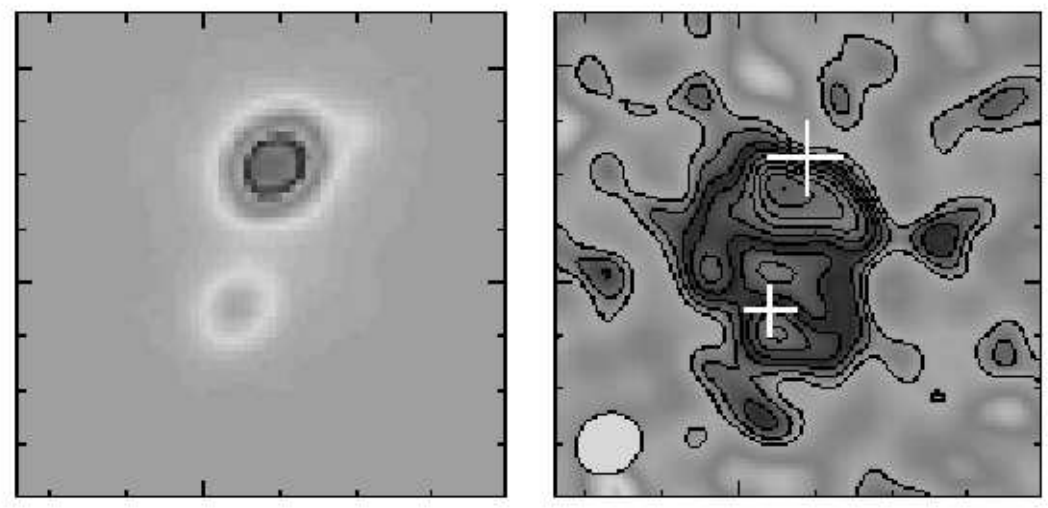

Figure 3. The optical (left) and CO 2-1 (right) images of the gravitationally lensed QSO J2322+1944 at $z=4.12$ (Carilli et al. 2003).

Figure 3 shows the optical and CO emission from the $z=4.12$ QSO $2322+1944$. The source is strongly gravitationally lensed, appearing as a double QSO in the optical with 1" separation (Djorgovski et al. in prep), and a complete 'Einstein ring' in the CO 2-1 emission. A similar ring is seen for the $1.4 \mathrm{GHz}$ continuum emission (Carilli et al. 2003). These results can be modeled in the source plane as a starburst disk surrounding the QSO with a radius of about $2 \mathrm{kpc}$. J2322+1944 represents perhaps the best example of a coeval starburst + AGN at high redshift.

We consider briefly the question of why only $30 \%$ of high $z$ QSOs are FIR luminous? Unfortunately, sensitivity limits of current (sub)mm surveys cannot rule out a continuum of FIR luminosities extending below a few mJy, although deep radio surveys suggest that this may not be the case (Petric et al. 2004 in prep). If there really are two types of QSOs (FIRluminous and FIR-quiet), then the $30 \%$ fraction could represent a relative duty cycle for star formation vs. QSO activity. Alternatively, there may be 
two types of major accretion events for SMBHs: those that have associated star formation, and those that do not.

\section{Cosmic Stromgren spheres}

The epoch of reionization (EoR) represents a fundamental benchmark in cosmic structure formation, corresponding to ionization of the neutral IGM by the first luminous sources. The recent discovery of Ly $\alpha$ absorption by the neutral IGM toward $z>6$ QSOs (the Gunn-Peterson effect; Fan et al. 2003) implies that these sources are situated at the end of the EoR.
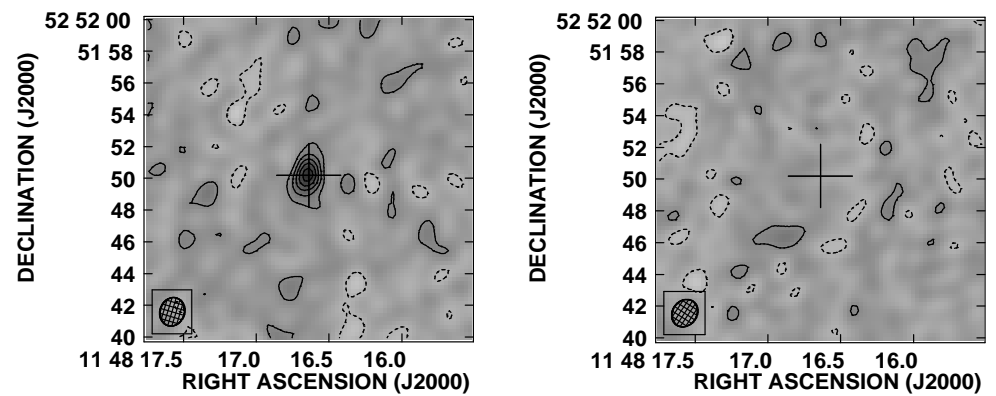

Figure 4. CO 3-2 emission from the highest redshift QSO, J1148+5251 at $z=6.419$ (Walter et al. 2003). The left frame shows the velocity integrated line emission at $1.5^{\prime \prime}$ resolution at $46.7 \mathrm{GHz}$. The right frame is an 'off' channel, showing the lack of continuum emission. The contour levels are: - $0.1,0.1,0.2,0.3,0.4,0.5 \mathrm{mJy} / \mathrm{beam}$.

Bertoldi et al. (2003) show that the $30 \%$ fraction of FIR-luminous QSOs remains constant into the EoR, including the most distant QSO known, J1148+5251, at $z=6.42$, which has an $L_{F I R}=1.2 \times 10^{13} \mathrm{~L}_{\odot}$. CO emission has also been detected from J1148+5251 using the VLA and the PdBI (Figure 4; Walter et al. 2003; Bertoldi et al. 2003), with an implied molecular gas mass of $2 \times 10^{10} \mathrm{M}_{\odot}$. The presence of a large mass of heavy elements and dust in a galaxy just 0.8 Gyrs from the big bang raises interesting issues for ISM enrichment, and suggests that active star formation started at $z>10$ in the host galaxy of this QSO. Also required is a dust formation mechanism involving high mass stars and/or supernova remnants.

A particularly interesting result for $\mathrm{J} 1148+5251$ is the difference between the host galaxy redshift of 6.42 (as determined from the CO line) 
and the on-set of the Gunn-Peterson absorption trough at $z=6.32$. We note these $\mathrm{CO}$ observations are the only measurements relating to the host galaxy properties (eg. the exact redshift), as opposed to the AGN properties. This redshift difference implies that the QSO must be surrounded by a ionized sphere of physical radius $\mathrm{R}=4.7 \mathrm{Mpc}$, presumably formed by the radiation from the QSO itself. Hence, in J1148+5251 we are witnessing the process of cosmic reionization. This is a 'time bounded' Stromgren sphere, and implies a lifetime for the recent QSO activity of: $\mathrm{t}_{\mathrm{qso}}=10^{7}(\mathrm{R} / 4.5 \mathrm{Mpc})^{3} \mathrm{f}(\mathrm{HI})$ years, where $\mathrm{f}(\mathrm{HI})$ is the (volume averaged) IGM neutral fraction (Walter et al. 2003; Wyithe \& Loeb 2003a).

Wyithe \& Loeb (2003b) have used this result, plus models of QSO formation, to argue (statistically) that the neutral fraction of the IGM must be substantial at $z=6.4: \mathrm{f}(\mathrm{HI})>0.1$, otherwise the QSO lifetimes become unreasonably short. This neutral fraction is much larger than the best current lower limits set by the GP effect of $\mathrm{f}(\mathrm{HI})>0.001$, and argues for 'fast reionization' at $z \sim 6$ (Gnedin 2000). The implication is that $\mathrm{f}(\mathrm{HI})$ changes from $<10^{-4}$ at $z=5.7$ to $>10^{-1}$ at $z=6.4$, ie. the neutral fraction of the universe changes by three orders of magnitude over a timescale of only 0.1 Gyr.

\section{References}

1. Alloin, D. et al. 2000, ApJ, 528, L81

2. Baker, A. et al. 2004, astroph-

3. Barvainis, R. 1999, in Highly Redshifted Radio Lines, (ASP: San Francisco), eds. Carilli et al. p. 39

4. Barvainis, R. et al. 1998, ApJ, 492, L13

5. Barvainis, R. et al. 2002, A\&A, 385, 399

6. Beelen, A. et al. 2004, A\&A, submitted

7. Beelen, A. et al. 2004, A\&A, in prep

8. Bertoldi, F. et al. 2003, A\& A, 409, L47

9. Bertoldi, F. et al. 2003, A\& A, 406, L55

10. Carilli, C. et al. 2003, Science, 300, 773

11. Carilli, C. et al. 2002, AJ, 123, 1838

12. Cox, P. et al. 2002, A\&A, 387, 406

13. Downes, D. \& Solomon, P. 1998, ApJ, 507, 615

14. de Breuck, C. et al. 2003, NewAR, 47, 285

15. Fan, X. et al. 2003, AJ, 125, 1649

16. Frayer, D. et al. 1999, ApJ, 514, L13

17. Gao, Y. \& Solomon, P. 2003, ApJ (in press) astroph0310339

18. Gebhardt, K. et al. 2000, ApJ, 539, L13

19. Genzel, R. et al. 2003, ApJ, 584, 633

20. Gnedin, N. 2000, ApJ, 535, 530 
21. Greve, T., Ivison, R., Papadopoulos, P. 2004, A\&A, in press

22. Guilloteau, S. et al. 1999, A\&A, 349, 363-368

23. Neri, R. et al. 2003, 597, L113

24. Omont, A. et al 2003, A\&A, 398, 857

25. Papadopoulos, P. et al. 2001, Nature, 409, 58

26. Papadopoulos, P. et al. 2000, ApJ, 528, 626

27. Sanders, D. \& Mirabel, F. 1996, ARAA, 34, 749

28. Scoville, N. et al. 2003, ApJ, 585, L105

29. Solomon, P. et al. 2003, Nature, 426, 636

30. Solomon, P., Downes, D., \& Radford, S. 1992 Nature, 356, 318

31. Walter, F. et al. 2003, Nature, 424, 406

32. Weiss, A. et al. A\&A, 409, L41

33. Wyithe, S. \& Loeb, A. 2004a, astro-ph/0401188

34. Wyithe, S. \& Loeb, A. 2004b, astro-ph/0401554

35. Yu, Q. \& Tremaine, S. 2002, MNRAS, 335, 965 